\title{
Efeitos do ozônio nas lesões de reperfusão do jejuno em eqüinos
}

[Effects of ozone in equine jejunum reperfusion injury]

\author{
G.E.S. Alves ${ }^{1}$, J.M.G. Abreu ${ }^{2}$, J.D. Ribeiro Filho ${ }^{3}$, L.A.L. Muzzi ${ }^{4}$, \\ H.P. Oliveira ${ }^{1}$, R.J. Tannus ${ }^{5}$, T. Buchanan ${ }^{6}$ \\ ${ }^{1}$ Escola de Veterinária - UFMG \\ Caixa Postal 567 \\ 30123-970 - Belo Horizonte, MG \\ ${ }^{2}$ Universidade Estadual do Ceará - Fortaleza, CE \\ ${ }^{3}$ Universidade Federal de Viçosa - Viçosa, MG \\ ${ }^{4}$ Universidade Federal de Lavras - Lavras, MG \\ ${ }^{5}$ Faculdade de Jaguariúna - SP \\ ${ }^{6}$ Brazos Valley Equine Hospital Inc. - Texas (EUA)
}

\begin{abstract}
RESUMO
Investigaram-se os efeitos do ozônio nas lesões de reperfusão intestinais de eqüinos. Induziu-se obstrução vascular (2h) seguida de reperfusão (12h) e os animais receberam os seguintes protocolos: não tratado $(n=7,500 \mathrm{ml}$ solução salina $0,9 \%)$ e tratado com ozônio $\left(n=6,50 \mu \mathrm{gkg}^{-1}\right)$. Amostras intestinais foram examinadas em $0,1,2 \mathrm{~h}$ (obstrução) e 1, 2, 12h (reperfusão). Os seguintes escores histomorfológicos apresentaram-se significativamente atenuados: na região da mucosa - desprendimento epitelial, infiltrado de neutrófilos e hemorragia; na submucosa - infiltrado de neutrófilos e edema. Essas diferenças ocorreram na fase inicial da reperfusão, coincidindo com a geração de radicais livres derivados do oxigênio. Os efeitos conservadores observados podem estar associados à modulação de enzimas antioxidantes, ou à propriedades bioquímicas do ozônio, que interferiram com etapas bioquímicas da reperfusão, representando uma alternativa terapêutica para o tratamento de pacientes acometidos por abdome agudo.
\end{abstract}

Palavras-chave: eqüino, ozônio, reperfusão, jejuno

\begin{abstract}
This study was designed to investigate the effects of ozone on attenuating jejunum reperfusion injury in horses. Vascular obstruction(2h)/reperfusion $(12 \mathrm{~h})$ was induced and the animals received the following protocols: not treated $\left(n=7,500 \mathrm{ml}\right.$ saline solution) or treated with ozone $\left(n=6,50 \mu \mathrm{g}^{\mathrm{kg}} \mathrm{kg}^{-1}\right)$. Intestinal samples were histomorphologically examined at 0, 1, $2 \mathrm{~h}$ (obstructive) and 1, 2, $12 \mathrm{~h}$ (reperfusion phase). The following scores were significantly attenuated: mucosal region-epithelial loss, neutrophil infiltration and hemorrhage; submucosal region-neutrophil infiltration and edema. These effects may be related to the antioxidant enzyme modulation, or ozone biochemical properties, which interfered with biochemical steps of reperfusion injury. The results indicate an alternative for the treatment of equine acute abdomen.
\end{abstract}

Keywords: equine, ozone, reperfusion, jejunum

\section{INTRODUÇÃO}

O trato gastrintestinal é particularmente dotado de recursos enzimáticos para formação de grande concentração de radicais livres derivados do oxigênio (RLO) e há evidências de formação desses agentes no intestino delgado de eqüinos (Prichard et al., 1991).

Recebido para publicação em 2 de abril de 2003

Recebido para publicação, após modificações, em 28 de junho de 2004

E-mail: geraldo@vet.ufmg.br 
A terapia antioxidante constitui uma nova abordagem farmacológica na inflamação e lesão de isquemia e reperfusão (Cuzzocrea et al., 2001). Trabalhos anteriores relataram que a manutenção e o incremento de sistemas antioxidantes induzidos por ozônio $\left(\mathrm{O}_{3}\right)$ atenuaram as lesões induzidas pelos RLO (Hernandez et al., 1995; Leon et al., 1998; Peralta et al., 1999). $\mathrm{O} \mathrm{O}_{3}$ mostrou-se eficiente em diversas enfermidades circulatórias e inflamatórias (Shiratori et al., 1993; Bocci, 1994; Bulmer et al., 1997), e a exposição sangüínea a baixas concentrações contribuiu para a proteção das células ao estresse oxidativo (Ploppler et al., 1994; Ajamieh et al., 2002).

Em pesquisa com células endoteliais humanas, constatou-se elevação significativa da concentração de óxido nítrico liberado, que é um modulador da vasodilatação (Valacchi e Bocci, 2000). Em doença arterial oclusiva periférica, verificaram-se melhoras nas propriedades hemorreológicas, em pacientes sob ozônioterapia (Giunta et al., 2001). O presente estudo teve o objetivo de avaliar os efeitos do ozônio nas lesões de reperfusão do jejuno em eqüinos.

\section{MATERIAL E MÉTODOS}

Foram usados 13 eqüinos adultos, distribuídos em dois grupos: não tratado (G-NTR) e tratado com ozônio (G-OZO). Após a anestesia, os animais foram submetidos a dois modelos de obstrução (OBS) vascular simultânea, hemorrágico e isquêmico, pela interrupção da circulação mural, venosa (OVEN) ou artériovenosa (OARV) com drenos de Penrose. Um terceiro segmento não submetido à interrupção do fluxo sangüíneo, permaneceu como controle (CONT). Após duas horas, a circulação foi restabelecida, pela retirada das ligaduras, quando se iniciou a fase de reperfusão (REP), com duração de 12 horas. Os eqüinos do G-NTR receberam $500 \mathrm{ml}$ de soro fisiológico e os do G$\mathrm{OZO}, 500 \mathrm{ml}$ de soro ozonizado ${ }^{1}$ na dose de $50 \mu \mathrm{gkg}^{-1}$, ambos ao final da fase de obstrução vascular. As amostras foram colhidas antes da aplicação das ligaduras ( $0 \mathrm{~h})$, e nos tempos de $1 \mathrm{~h}$ e $2 \mathrm{~h}$ na fase de OBS, e $1 \mathrm{~h}, 2 \mathrm{~h}$ e $12 \mathrm{~h}$ na fase de REP. Após fixação em formalina neutra $10 \%$ tamponada, foram processadas pela técnica de inclusão em parafina e coradas por hematoxilinaeosina (Luna, 1968).

Os escores histomorfológicos foram classificados utilizando-se os critérios de Chiu et al. (1970) e Sullins et al. (1985). As comparações entre os dois grupos, em cada tempo, por segmento, em relação às medidas dos escores, foram realizadas utilizando-se o teste de Kruskal-Wallis, considerando-se significativos em $\mathrm{P}<5 \%$.

\section{RESULTADOS}

Os resultados dos escores médios e medianos das variáveis estudadas são apresentados nas Tab. de 1 a 5.

A caracterização microscópica das alterações nos segmentos intestinais obstruídos variou em intensidade, em ambas as fases do experimento. Houve graus variáveis de desprendimento epitelial, formação de fendas subepiteliais e vacuolização do epitélio de absorção. A perda epitelial iniciou-se nas extremidades das vilosidades, progredindo em direção à região das criptas. Ocorreu dilatação cística das criptas e acúmulos multifocais de debris na superfície da lâmina própria. As vilosidades tornaram-se encurtadas e espessadas. Edema, congestão capilar e hemorragia foram freqüentes na lâmina própria e infiltrado de neutrófilos foi observado na mucosa e submucosa. A túnica muscular foi minimamente afetada.

O restabelecimento da circulação exacerbou os escores histológicos, com tendência para maior ruptura da superfície da mucosa, edema, hemorragia e infiltrado de neutrófilos.

No G-OZO, observou-se atenuação significativa dos seguintes escores: no segmento venoso - na mucosa, desprendimento epitelial (1h REP), hemorragia (2h REP) e infiltrado de neutrófilos (1h REP e $2 \mathrm{~h}$ REP) e na submucosa, infiltrado de neutrófilos (1h REP) e edema (2h REP); no segmento artério-venoso - na mucosa, infiltrado de neutrófilos (1h REP e $2 \mathrm{~h}$ REP) e na submucosa, infiltrado de neutrófilos (1h REP e 2h REP) e edema (2h REP).

\footnotetext{
${ }^{1}$ Gerador de ozônio VM-45 Ven-Mas Scientific, Inc.-EUA
} 
Efeitos do ozônio nas les̃̃es...

Tabela 1. Escores medianos e médios para desprendimento epitelial na mucosa nos grupos experimentais e segmentos intestinais

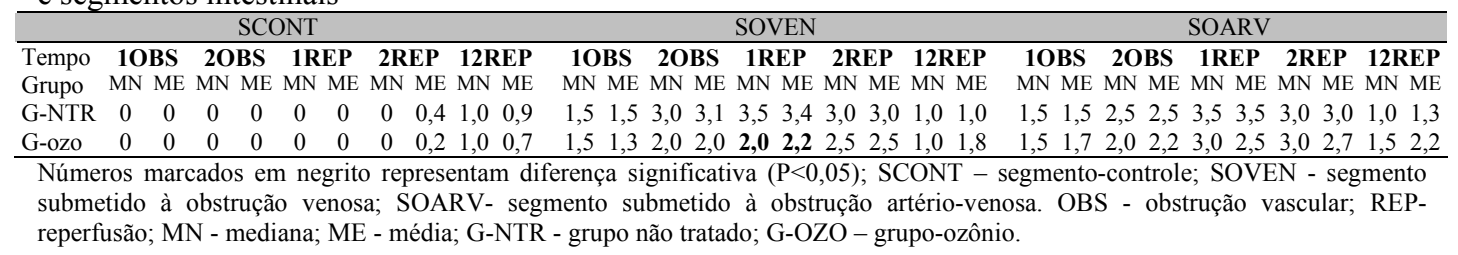

Tabela 2. Escores medianos e médios de hemorragia na mucosa nos grupos experimentais e segmentos intestinais

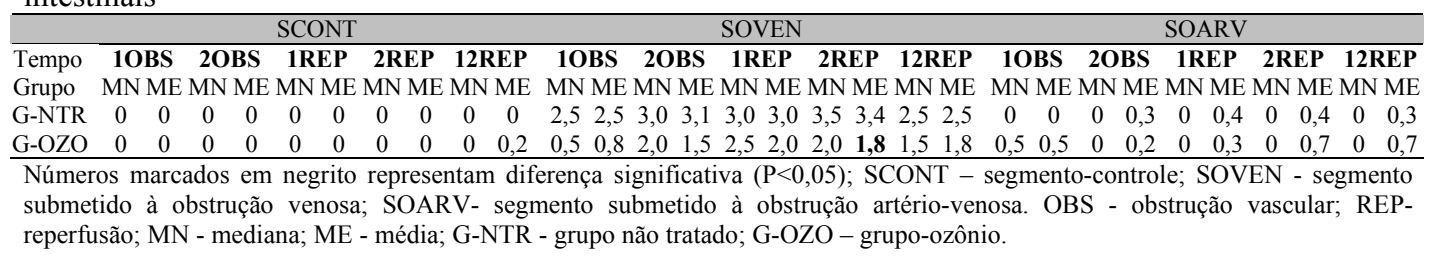

Tabela 3. Escores medianos e médios de neutrófilos na mucosa nos grupos experimentais e segmentos intestinais

\begin{tabular}{l}
\hline \multicolumn{1}{c}{ SCONT } \\
\hline Tempo 1OBS
\end{tabular}

Tabela 4. Escores medianos e médios de neutrófilos na submucosa nos grupos experimentais e segmentos intestinais

\begin{tabular}{|c|c|c|c|c|c|c|c|c|c|}
\hline & \multicolumn{7}{|c|}{ SCONT } & SOVEN & SOARV \\
\hline Tempo & 10 & BS & 20 & BS & 1REP & 2REP & 12REP & 1OBS 2OBS 1REP 2REP 12REP & 1OBS 2OBS 1REP 2REP 12REP \\
\hline Grupo & MN & $\mathrm{ME}$ & $\mathrm{MN}$ & $\mathrm{ME}$ & MN ME I & MN ME & MN ME & MN ME MN ME MN ME MN ME MN ME & MN ME MN ME MN ME MN ME MN ME \\
\hline G-NTR & 0 & 0 & 0 & 0 & 0 & 0 & 00 & $\begin{array}{llllllllll}0,5 & 0,6 & 0,5 & 0,6 & 2,0 & 2,0 & 2,0 & 2,0 & 1,5 & 1,4\end{array}$ & $\begin{array}{lllllllll}0 & 1,0 & 0,6 & 2,0 & 2,0 & 1,5 & 1,5 & 0,5 & 0,5\end{array}$ \\
\hline $\mathrm{G}-\mathrm{OZO}$ & 0 & 0 & 0 & 0 & 0 & 0 & 0 & $\begin{array}{llllllllll}1,0 & 1,0 & 1,0 & 1,0 & \mathbf{1 , 0} & 0,8 & 1,0 & 1,3 & 1,0 & 1,2\end{array}$ & $\begin{array}{llllllllll}0,5 & 0,5 & 0 & 0,3 & \mathbf{0} & 0,5 & \mathbf{0 , 5} & 0,5 & 1,0 & 0,8 \\
\end{array}$ \\
\hline
\end{tabular}

Tabela 5. Escores medianos e médios de edema na submucosa nos grupos experimentais e segmentos intestinais

\begin{tabular}{|c|c|c|c|c|c|c|c|c|c|c|c|c|c|c|c|c|c|c|c|c|c|c|c|c|c|c|c|}
\hline & \multicolumn{10}{|c|}{ SCONT } & \multicolumn{10}{|c|}{ SOVEN } & \multicolumn{7}{|c|}{ SOARV } \\
\hline Tempo & 10 & & & $\overline{\mathbf{B S}}$ & 1R & EP & $2 R$ & & $12 R$ & EP & 101 & & 201 & BS & $\overline{1 R Y}$ & EP & $2 \mathbf{2 R}$ & EP & $12 R$ & EP & 10 & $\mathrm{BS}$ & 20 & $\mathrm{BS}$ & 1REP & 2REP & 12REP \\
\hline \multirow{2}{*}{ Grupo } & M & M & M & M & $\mathrm{M}$ & M & M & M & $\mathrm{M}$ & $\mathrm{M}$ & M & $\mathrm{M}$ & M & M & M & M & M & $\mathrm{M}$ & M & M & M & M & M & M & M M & M M & M M \\
\hline & $\mathrm{N}$ & E & $\mathrm{N}$ & E & $\mathrm{N}$ & $\mathrm{E}$ & $\mathrm{N}$ & E & $\mathrm{N}$ & E & $\mathrm{N}$ & E & $\mathrm{N}$ & E & $\mathrm{N}$ & $\mathrm{E}$ & $\mathrm{N}$ & $\mathrm{E}$ & $\mathrm{N}$ & $\mathrm{E}$ & $\mathrm{N}$ & $\mathrm{E}$ & $\mathrm{N}$ & $\mathrm{E}$ & $\mathrm{N}$ & $\mathrm{N}$ & $\mathrm{N}$ \\
\hline G-NTR & 0 & 0 & 0 & 0 & 0 & 0 & 0 & 0 & 0 & 0 & 1,5 & 1,4 & 2,5 & 2,4 & 3,0 & 2,8 & 2,5 & 2,7 & 2,0 & 1,6 & 0 & 0 & 0 & 0,3 & $1,0 \quad 0,7$ & $1,0 \quad 1,1$ & $1,0 \quad 0,7$ \\
\hline G-OZO & 0 & 0 & 0 & 0 & 0 & 0 & 0 & 0 & 0 & 0 & 1,0 & 1,2 & 2,0 & 1,7 & 2,0 & 2,0 & 1,5 & 1,5 & 1,5 & 1,7 & 0 & 0,2 & 0 & 0,2 & $1,0 \quad 1,0$ & $1,0 \quad 0,8$ & $1,0 \quad 1,2$ \\
\hline
\end{tabular}

\begin{tabular}{llllllllllllllllllllllllllllllllllll}
$\mathrm{G}-\mathrm{OZO}$ & 0 & 0 & 0 & 0 & 0 & 0 & 0 & 0 & 0 & 0 & 1,0 & 1,2 & 2,0 & 1,7 & 2,0 & 2,0 & $\mathbf{1 , 5}$ & 1,5 & 1,5 & 1,7 & 0 & 0,2 & 0 & 0,2 & 1,0 & 1,0 & 1,0 & 0,8 & 1,0 & 1,2 \\
\hline
\end{tabular} submetido à obstrução venosa; SOARV- segmento submetido à obstrução artério-venosa. OBS - obstrução vascular; REPreperfusão; MN - mediana; ME - média; G-NTR - grupo não tratado; G-OZO - grupo-ozônio. 


\section{Alves et al.}

\section{DISCUSSÃO}

Diversas pesquisas já foram realizadas buscando drogas com potencial para atenuar a lesão de reperfusão. Embora vários agentes e procedimentos estejam disponíveis, nenhum é plenamente eficaz e utilizado na rotina. Novas estratégias farmacológicas de intervenção necessitam melhor avaliação em medicina veterinária, como as que usam agentes antioxidantes (Cuzzocrea, 2001). O maior número de experimentos que utilizou $\quad \mathrm{O}_{3}$ avaliou o seu efeito oxidante. A produção de RLO é considerada a principal causa de lesão tecidual, durante a reperfusão. Em condições patológicas, os mecanismos de defesa enzimáticos podem ser superados, permitindo que os radicais exerçam efeitos deletérios. Já foi comprovado, no jejuno de eqüinos, o potencial bioquímico para a formação desses agentes, via cascata da xantina oxidase (Prichard et al., 1991). Nos segmentos de jejuno estudados ocorreu lesão de reperfusão, comprovada pelo agravamento das lesões na fase de pós-obstrução vascular, no grupo não tratado. A lesão foi progressiva e consistiu de congestão, edema, infiltrado de neutrófilos, hemorragia, e necrose da mucosa.

Demonstrou-se a eficiência do $\mathrm{O}_{3}$, visto que ocorreu atenuação de diversos escores histomorfológicos. As alterações que acompanham o processo de inflamação e necrose local, quando exacerbadas, podem contribuir para o agravamento da lesão na mucosa. Clinicamente, a proteção tecidual no início da reperfusão é importante, pois lesões extensas favorecem a passagem de bactérias e endotoxinas para circulação sistêmica, podendo desencadear quadros irreversíveis em pacientes com abdome agudo. Neste experimento, buscou-se o efeito paradoxal do $\mathrm{O}_{3}$ já relatado em diversos trabalhos (Plopper et al., 1994; Leon, 1998; Peralta et al., 1999). Esses autores descreveram que $\mathrm{o} \mathrm{O}_{3}$, por ser um oxidante, promove o estresse oxidativo e induz elevação dos sistemas enzimáticos antioxidantes para atenuação da lesão de reperfusão.

Giunta et al. (2001) relataram, em doença arterial oclusiva, após uma única dose de autohemoterapia ozonizada, melhora das propriedades reológicas do sangue e oxigenação dos tecidos, o que pode ser um mecanismo de atuação no atual experimento. Valacchi e Bocci
(2000) demonstraram que células endoteliais tratadas com o agente aumentaram a liberação de óxido nítrico, induzindo vasodilatação em áreas isquêmicas e reduzindo a hipóxia. Os mesmos autores verificaram inibição de moléculas de aderência, o que pode diminuir o processo inflamatório local. Como o $\mathrm{O}_{3}$ é um gás muito reativo e instável, pode-se prever que seu mecanismo de ação esteja relacionado diretamente com os produtos que ele gera, através de interações seletivas com compostos orgânicos que estão presentes no plasma e nas membranas celulares. Há evidências para afirmar que grande parte da lesão de isquemia e reperfusão intestinal seja o resultado de respostas inflamatórias causadas por produtos oxidativos de neutrófilos residentes ou infiltrados. Neste trabalho, verificou-se atenuação dos escores de neutrófilos, o que contribuiu para preservação da mucosa.

A homeostase do cálcio está alterada durante a isquemia em virtude da perda da atividade da bomba transportadora, o que permite a elevação da sua concentração intracelular, ativando enzimas como fosfolipases e endonucleases. Ajamieh et al. (2002) afirmaram que o efeito protetor do $\mathrm{O}_{3}$ na homeostase do cálcio deveu-se à conservação das atividades de ATPases- $\mathrm{Ca}^{2+}$. Essa situação pode ter ocorrido nas condições do atual experimento, contribuindo para graus de lesões mais discretos na reperfusão. Ainda, é possível que tenha ocorrido manutenção do metabolismo energético local, contribuindo para a preservação da mucosa, como verificado por Shiratori et al. (1993).

Diversos autores recomendaram o $\mathrm{O}_{3}$ em doses de $1 \mathrm{mg} . \mathrm{kg}^{-1}$ por 10 dias (Leon et al., 1998; Ajamieh et al., 2002). Dessa forma, o fenômeno de pré-condicionamento oxidativo é alcançado, sem toxicidade apreciável. Neste trabalho, a dose única aplicada foi de $50 \mu \mathrm{gkg}^{-1}$. Os efeitos benéficos constatados, mesmo com a dose reduzida, provaram a eficiência da droga, sendo possível que doses superiores ou adicionais possam exercer melhor e mais duradouro efeito, prolongando-se até a fase de regeneração intestinal.

Concluiu-se que o $\mathrm{O}_{3}$ é eficaz na atenuação de lesão de reperfusão no jejuno de eqüinos submetidos à obstrução vascular. A proteção pode ter decorrido por efeitos hemorreológicos, 
hemodinâmicos sistêmicos ou modulatório sobre enzimas antioxidantes.

\section{REFERÊNCIAS BIBLIOGRÁFICAS}

AJAMIEH, H.; MERINO, N.; CANDELARIOJALIL, E. et al. Similar protective effect of ischaemic and ozone oxidative preconditionings in liver ischaemia/reperfusion injury. Pharmacol. Res., v.45, p.333-339, 2002.

BULMER, J.; BOLTON, A.E.; POCKLEY, A.G. Effect of combined heat, ozonation and ultraviolet irradiation (Vasocare) on heat shock protein expression by peripheral blood leukocyte populations. J. Biol. Regul. Homeost. Agents, v.11, p.104, 1997.

CHIU, C.J.; MCARDLE, A.H.; BROWN, R. et al. The intestinal mucosa lesion in low flow states: a morphologic, hemodynamic and metabolic reappraisal. Arch. Surg., v.101, p.478, 1970.

CUZZOCREA, S.; RILEY, D.P.; CAPUTI, A.P. et al. Antioxidant therapy: a new pharmacological approach in shock, inflammation, and ischemia/reperfusion injury. Pharmacol. Rev., v.53, p.135-159, 2001.

GIUNTA, R.; COPPOLA, A.; SAMMARTINO, A. et al. Ozonized autohemotransfusion improves hemorheological parameters and oxygen delivery to tissues in patients with peripheral occlusive arterial disease. Ann. Hematol., v.80, p.745-748, 2001.

HERNANDEZ, F.; MENENDEZ, S.; WONG, R. Decrease of blood cholesterol and stimulation of antioxidative response on cardiopathy patients treated with endovenous ozone therapy. Free Rad. Biol. Med., v.19, p.115-119, 1995.

LEON, O.S.; MENENDEZ, S.; MERINO, N. et al. Ozone oxidative preconditioning: a protection against cellular damage by free radicals. Mediators Inflam., v.7, p.289-294, 1998.

LUNA, L.G. Manual of histologic staining methods of the arms force institute of pathology. 3.ed. New York: McGraw Hill, 1968.

PERALTA, C.; LEON, O.S.; XAUS, C. et al. Protective effect of ozone treatment on the injury associated with hepatic ischemia-reperfusion: antioxidant-prooxidant balance. Free Rad. Res., v.31, p.191-196, 1999.

PLOPPER, C.G.; DUAN, X.; BUCKPITT, A.R. et al. Dose dependent tolerance of ozone. Toxicol. Appl. Pharmacol., v.127, p.124-131, 1994.

PRICHARD, M.; DUCHARME, N.G.; WILKINS, P.A. et al. Xanthine oxidase formation during experimental ischemia of the equine small intestine. Can. J. Vet Res., v.55, p.310-314, 1991.

SHIRATORI, R.; KANEKO, Y.; YAMAMOTO, Y. et al. Can ozone administration activate tissue metabolism? A study on brain metabolism during hipoxic hipoxia. Masui, v. 42, p. 2-6, 1993.

SULLINS, K.E.; STASHAK, T.S.; MERO, K.N. Pathologic changes associated with induced small intestinal strangulation obstruction and nonstrangulating infarction in horses. Am. J. Vet. Res., v.46, p.913-916, 1985.

VALACCHI, G.; BOCCI, V. Studies on the biological effects of ozone: 11. Release of factors from human endothelial cells. Mediators Inflam., v.9, p.271-276, 2000. 